\title{
Meningocele of the Internal Auditory Canal Requiring Facial-Nerve Decompression
}

\author{
Jeffrey Liaw Huseyin Isildak
}

Division of Otolaryngology - Head and Neck Surgery, Department of Surgery,

The Pennsylvania State University College of Medicine, Hershey, PA, USA

\section{Established Facts}

- Vestibular schwannoma and meningioma represent the vast majority of internal auditory canal (IAC) lesions.

- Due to the lack of bony covering between the cochlea and IAC, an IAC meningocele would raise concerns for a cerebrospinal fluid gusher and surgery is usually not recommended.

\section{Novel Insights}

- We report a case of an unruptured IAC meningocele resulting in facial-nerve paralysis.

- $\quad$ Surgical intervention should be pursued in cases with sudden facial paralysis.

\section{What Is It about?}

- In this case report, we describe a case of a 14-month-old boy with a cystic lesion of his left internal auditory canal (IAC) that resulted in sudden left-sided facial paralysis. Due to concerns regarding his facial-nerve function, he was taken to the operating room for facial-nerve decompression. Although preoperative MRI was performed due to suspicion of an epidermoid cyst, intraoperatively, no cystic lesion was identified. Tissue biopsied from the IAC demonstrated benign glial tissue consistent with a meningocele. Review of the literature demonstrates only one other case described of an IAC meningocele.

\section{Keywords}

IAC $\cdot$ Meningocele $\cdot$ Internal auditory canal

\section{Abstract}

In this case report, we present the case of a 14-month-old boy with a history of left facial palsy which developed at a very young age. CT of the temporal bone revealed a cystic lesion 
of the left petrous apex, and sedated auditory testing revealed a profound hearing loss on the same side. Following his first episode of left facial palsy, his symptoms nearly fully resolved and he was lost to follow-up. However, he was seen 5 months later due to recurrent and sudden left-sided facial paralysis. MRI was performed due to suspicion of an epidermoid cyst. The patient was subsequently taken to the operating room for facial-nerve decompression. Intraoperatively, no obvious cystic lesion was identified. Tissue biopsied from the internal auditory canal demonstrated benign glial tissue and fibrous tissue consistent with a meningocele.

(C) 2020 The Author(s)

Published by S. Karger AG, Basel

\section{Introduction}

Lesions of the internal auditory canal (IAC) are common, with vestibular schwannoma representing the majority of masses, i.e., up to $90 \%$ of cases. Meningiomas represent nearly all remaining cases $(10 \%)$. However, rare masses such as facial neurinomas, cavernomas, hemangiomas, epidermoid cysts, arachnoid cysts, and metastatic tumors within the IAC have also been reported in the literature [1]. Here, we present a case of a 14-month-old male with an IAC lesion resulting in facial paralysis and necessitating a facial-nerve decompression.

\section{Clinical Presentation}

A 14-month-old male presented with sudden left-sided facial paralysis and left sensorineural hearing loss. His facial paralysis was worked up for a Bell's palsy and he underwent an MRI which was unremarkable.

Due to continued symptoms, repeat MRI of the IAC was performed 3 months later which demonstrated poor visualization of the 7 th and 8th cranial nerve complex as well as widening of the IAC.

Based on discussions with neuroradiology, there was concern for poor development of the left cochlear and facial nerves, and he therefore underwent a CT scan of the temporal bones as well as a sedated ABR test of his hearing.

Sedated ABR testing demonstrated a normal right ear with a left ear unresponsive to evoked stimuli. CT of the temporal bone showed a cystic lesion of the left IAC with extension to the petrous segment. A repeat MRI was recommended.

Following this recommendation, the patient's facial paralysis nearly resolved and he was lost to follow-up. He presented 5 months later following an episode of recurrent left facial paralysis. MRI revealed a cystic mass with compression of cranial nerves 7 and 8 , the P2 segment of the ICA, and the left cerebellopontine angle (Fig. 1). Due to sudden facial paralysis, he was scheduled for facial-nerve decompression.

\section{Surgical Procedure and Postoperative Course}

After induction of general anesthesia, a left postauricular incision was designed down to the mastoid tip and a Palva flap was elevated. A mastoidectomy was begun with a cutting burr and the tegmen tympani was identified. The antrum was opened and the lateral canal and incus were identified and kept intact. The facial recess was opened and the facial nerves were carefully identified with the overlying bone intact. The identity of the facial nerve was confirmed with the nerve stimulator.

Attention was then turned to the external auditory canal. A tympanomeatal flap was elevated and the middle ear space was entered. The incudostapedial joint was noted to be intact and the middle ear was otherwise normal. The inferior canal was then drilled down to 
Biomedicine

Hub

Fig. 1. Preoperative T2 MRI demonstrates nonvisualization of the 7-8th nerve complex within the left internal auditory canal (IAC) and left cerebellopontine angle with widening of the IAC (white arrow), suggestive of a cystic mass displacing the nerve to the periphery.

Fig. 2. Intraoperative finding of meningocele in the left internal auditory canal. Final pathology demonstrated normal glial tissue confirming meningocele.

\begin{tabular}{l|l}
\hline Biomed Hub 2020;5:507420 \\
\hline DOI: 10.1159/000507420 & $\begin{array}{l}\text { @ 2020 The Author(s). Published by S. Karger AG, Basel } \\
\text { www.karger.com/bmh }\end{array}$ \\
\hline
\end{tabular}

Liaw and Isildak: Meningocele of the IAC
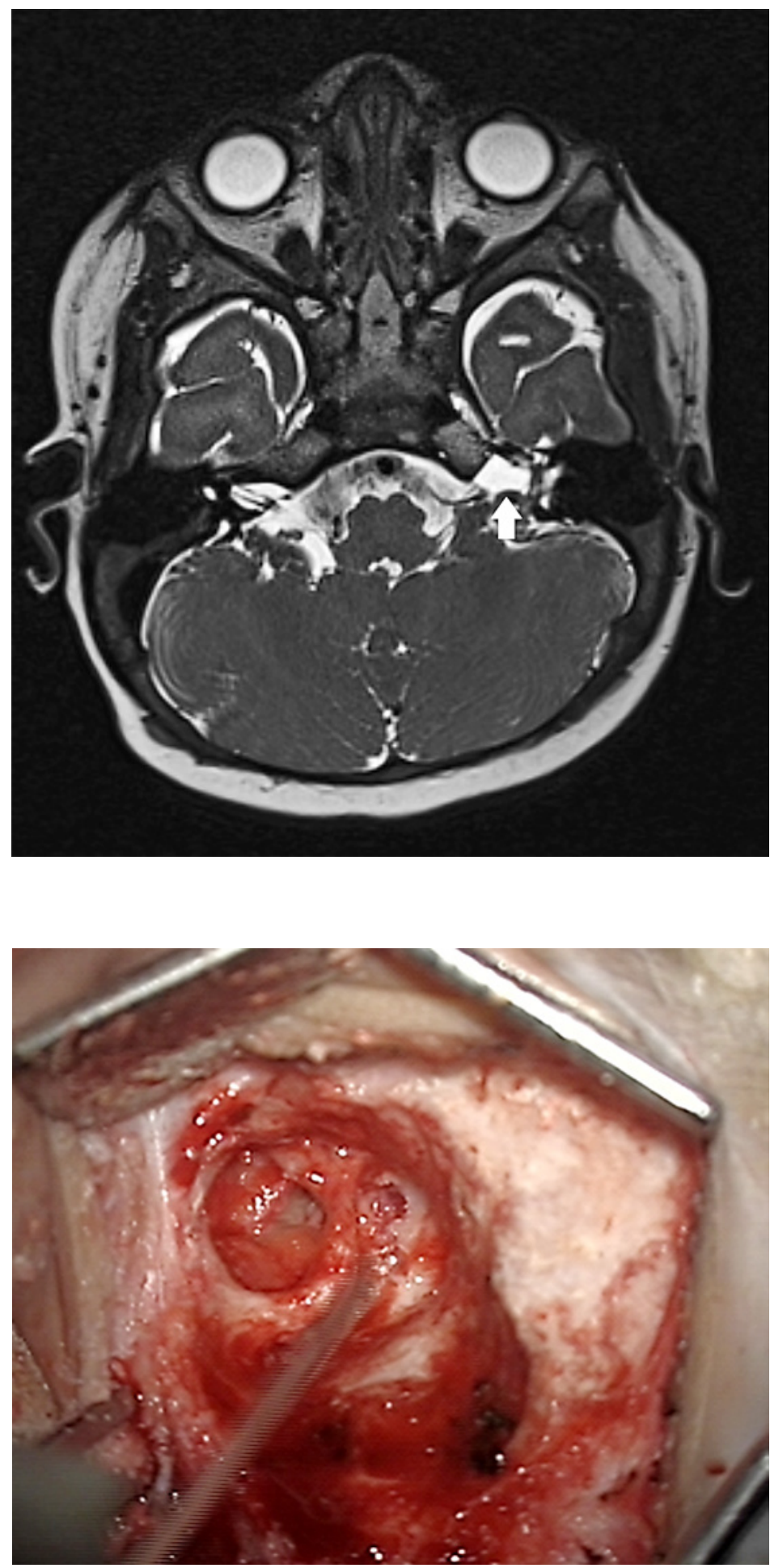

expose the hypotympanum. An infracochlear approach was used using the jugular bulb, round window, and carotid artery as the limits of the dissection. Some tissue was biopsied and sent to pathology for frozen section but no obvious cystic lesion was identified. The dura was opened in a small area during this dissection and leakage of cerebrospinal fluid (CSF) was noted. 
Fig. 3. Intraoperative frozen section of left petrous apex specimen demonstrating fragments of benign glial tissue and fibrous tissue consistent with meningocele. HE. $\times 200$.

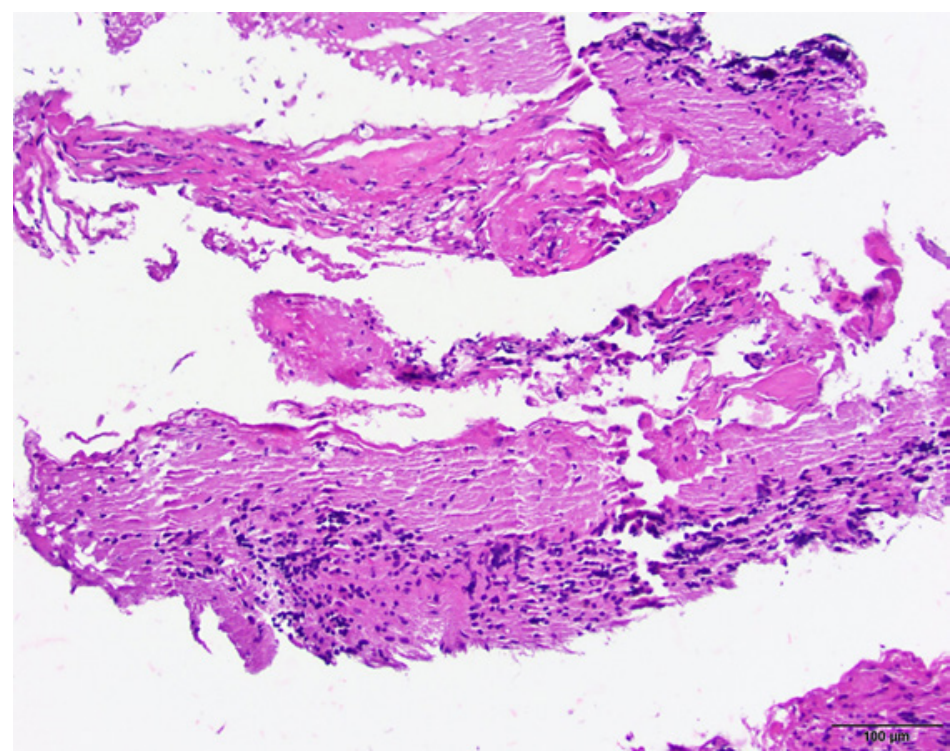

Attention was then turned to the mastoid. An infralabyrinthine approach was then performed to the petrous apex. The bone overlying the facial nerve was thinned posteriorly but kept intact. Again, no obvious cystic lesion was identified in the petrous apex (Fig. 2).

The decision was made to proceed with a translabyrinthine decompression. The canal wall was taken down. The anterior canal skin and tympanic membrane were removed. The malleus and incus were removed. Semicircular canals were opened and the stapes was removed. The cochlea was drilled down and the facial nerve was traced from the first genu to the IAC. The IAC was opened anteriorly, posteriorly, and inferiorly. Again, no cystic lesion was identified. However, the dura mater was seen. The facial nerve appeared irritated at the IAC and was deemed to be adequately decompressed in this region. Additional samples were taken for pathology. The dura mater was then covered with the temporal fascia and sealed with DuraSeal, the cavity packed with abdominal fat, and the Palva flap closed in layers.

Final histopathology of biopsies taken of the petrous apex demonstrated benign glial tissue highlighted on trichrome stain compatible with a meningocele/encephalocele (Fig. 3). During his postoperative visit at 4 months, the patient demonstrated continued improvement in his facial function with very mild weakness, graded as House Brackman II/VI. He was subsequently referred for a Baha Softband and follow-up at our Facial Nerve Clinic for continued monitoring. Postoperative MRI demonstrated successful decompression of the IAC meningocele and revisualization of the 7-8th nerve complex (Fig. 4).

\section{Discussion}

Although vestibular schwannomas and meningiomas represent the vast majority of IAC lesions, this case demonstrates that there are other rare lesions that may arise in the IAC with clinical consequences.

Imaging is therefore critical for distinguishing between the type of lesions in the IAC prior to any intervention. MRI is the imaging modality of choice for defining lesions in the cerebellopontine angle and IAC. In our case, MRI demonstrated an ill-defined cystic lesion centered in the left IAC and petrous apex isointense on T1 and T2 sequences without any significant enhancement. Marked dilation of the IAC with thinning of the otic capsule and erosion, and a 
Fig. 4. Postoperative T2 MRI demonstrating decompression of meningocele and revisualization of 7-8th nerve complex within the widened left internal auditory canal (white arrow).

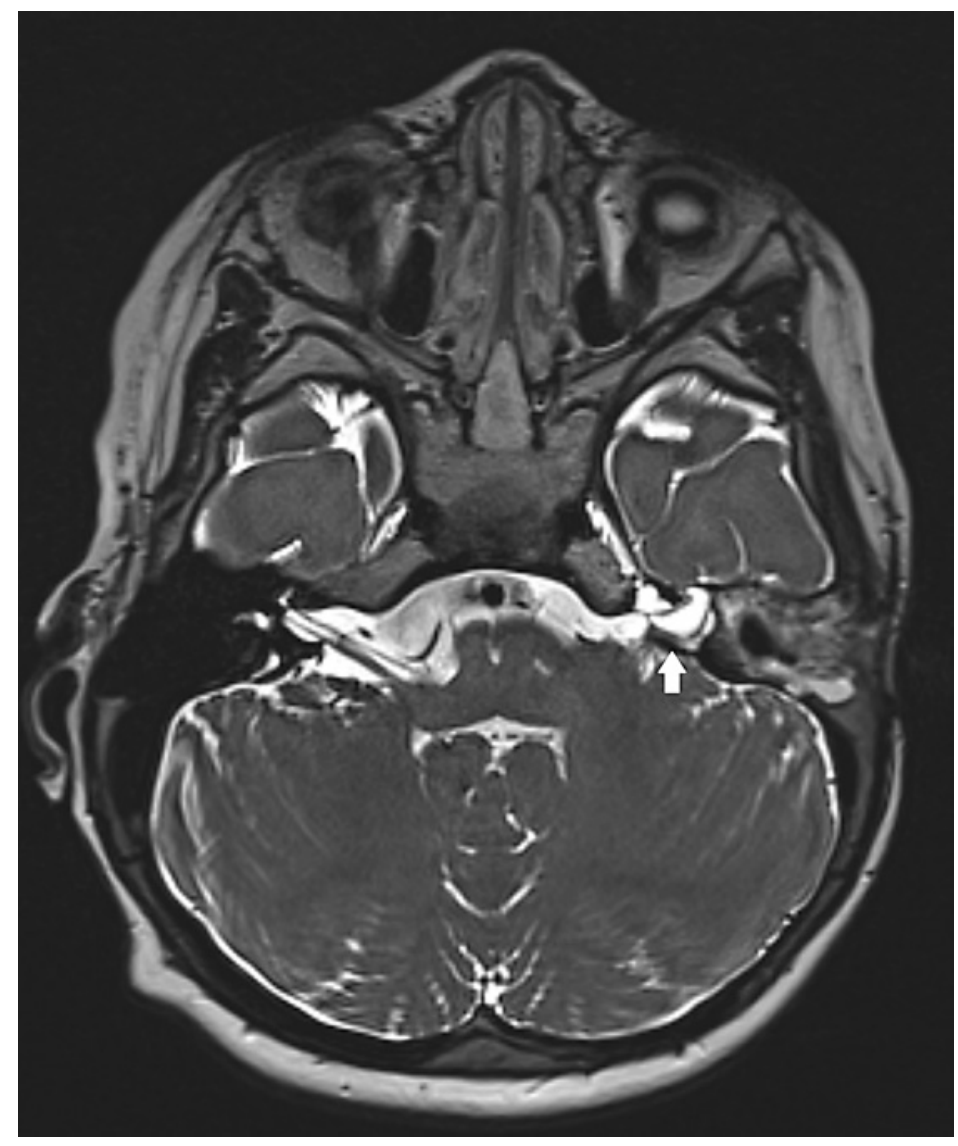

lack of bone covering between the cochlea and the IAC was visualized on CT. Genicular and tympanic segments of the facial nerve were also poorly delineated. The finding of profound sensorineural hearing loss with erosion of the otic capsule in a 14-month-old patient raised concerns for a congenital perilymphatic fistula. Congenital perilymphatic fistulas, however, are predominantly associated with middle-ear anomalies or CSF otorrhea [2]. Our patient had a radiologically normal middle ear with intact ossicles and had no rhinorrhea upon presentation.

The lack of bone covering between the cochlea and the IAC also raised concerns for pursuing surgical intervention, as communication between the perilymph in the cochlea and the IAC raises concerns of a CSF gusher [3]. CSF gushers may be difficult to control intraoperatively and may lead to postoperative meningitis. Our patient demonstrated progression of his meningocele which had resulted in sudden facial-nerve paralysis. Apicectomy and facialnerve decompression were pursued as the risk of permanent facial paralysis outweighed the risk of a CSF leak. The resulting CSF leak observed intraoperatively was successfully repaired by packing with abdominal fat and DuraSeal. The facial-nerve decompression within the IAC was successful in restoring the patient to House Brackmann grade II/VI.

The final pathology report findings of benign glial tissue supported the diagnosis of an IAC meningocele. An unruptured meningocele would also correlate with the lack of a CSF leak prior to intervention when compared to other clinical entities such as a translabyrinthine fistula [4].

Upon review of the literature, we found only one other case of an IAC meningocele; that particular patient presented with progressive left-sided hearing loss as his only symptom [5]. 
Due to this, surgery was not pursued and the case was presented as a reason not to pursue intervention. In contrast, our patient is the only case known in the literature of an IAC Meningocele in which surgery was pursued.

\section{Conclusion}

IAC meningoceles are rare lesions which have not been well described in the literature. The presence of an IAC meningocele can result in compression of the 7-8th nerve, and the lack of a bone covering between the IAC and cochlea can have implications for hearing and facial-nerve function. Surgical intervention for facial paralysis in an IAC meningocele may lead to a CSF leak. However, the risk of a CSF leak is outweighed by the benefits of the restoration of the function of the facial nerve.

\section{Statement of Ethics}

This study adheres to the tenets of the 1964 Declaration of Helsinki. The patient's parents provided informed consent for nonidentifiable medical imaging to be performed for scientific purposes.

\section{Disclosure Statement}

The authors have no conflicts of interest to declare.

\section{Author Contributions}

Dr. Liaw wrote and reviewed the manuscript. Dr. Isildak is the head Otorhinolaryngology surgeon and reviewed the manuscript. Both authors read, edited, and approved the case report.

\section{References}

1 Bonneville F, Sarrazin JL, Marsot-Dupuch K, Iffenecker C, Cordoliani YS, Doyon D, et al. Unusual lesions of the cerebellopontine angle: a segmental approach. Radiographics. 2001 Mar-Apr;21(2):419-38.

2 Weber PC, Perez BA, Bluestone CD. Congenital perilymphatic fistula and associated middle ear abnormalities. Laryngoscope. 1993 Feb;103(2):160-4.

3 Cabbarzade C, Sennaroglu L, Süslü N. CSF gusher in cochlear implantation: the risk of missing CT evidence of a cochlear base defect in the presence of otherwise normal cochlear anatomy. Cochlear Implants Int. 2015 Jul; 16(4):233-6.

4 Kong WK, Lee CH, Eunhye Y, Shin SH. Unruptured translabyrinthine meningocele without CSF otorrhea. Int ] Pediatr Otorhinolaryngol. 2014 Mar;78(3):566-9.

5 Truesdale CM, Peterson RB, Hudgins PA, Vivas EX. Internal auditory canal meningocele-perilabyrinthine/ translabyrinthine fistula: case report and imaging. Laryngoscope. 2016 Aug;126(8):1931-4. 